\title{
In Vitro Osteogenic Potential of Green Fluorescent Protein Labelled Human Embryonic Stem Cell-Derived Osteoprogenitors
}

\author{
Intekhab Islam, ${ }^{1}$ Gopu Sriram, ${ }^{2,3}$ Mingming Li, ${ }^{2}$ Yu Zou, ${ }^{2,3}$ Lulu Li, \\ Harish K. Handral, ${ }^{2}$ Vinicus Rosa, ${ }^{2}$ and Tong $\mathrm{Cao}^{2}$ \\ ${ }^{1}$ Oral and Maxillofacial Surgery, Faculty of Dentistry, National University of Singapore, Singapore \\ ${ }^{2}$ Oral Sciences, Faculty of Dentistry, National University of Singapore, Singapore \\ ${ }^{3}$ Experimental Dermatology Group, Institute of Medical Biology, Agency for Science, Technology and Research, Singapore \\ Correspondence should be addressed to Intekhab Islam; denii@nus.edu.sg
}

Received 5 July 2016; Accepted 27 October 2016

Academic Editor: Christian Dani

Copyright (C) 2016 Intekhab Islam et al. This is an open access article distributed under the Creative Commons Attribution License, which permits unrestricted use, distribution, and reproduction in any medium, provided the original work is properly cited.

\begin{abstract}
Cellular therapy using stem cells in bone regeneration has gained increasing interest. Various studies suggest the clinical utility of osteoprogenitors-like mesenchymal stem cells in bone regeneration. However, limited availability of mesenchymal stem cells and conflicting evidence on their therapeutic efficacy limit their clinical application. Human embryonic stem cells (hESCs) are potentially an unlimited source of healthy and functional osteoprogenitors (OPs) that could be utilized for bone regenerative applications. However, limited ability to track hESC-derived progenies in vivo greatly hinders translational studies. Hence, in this study, we aimed to establish hESC-derived OPs (hESC-OPs) expressing green fluorescent protein (GFP) and to investigate their osteogenic differentiation potential in vitro. We fluorescently labelled H9-hESCs using a plasmid vector encoding GFP. The GFP-expressing hESCs were differentiated into hESC-OPs. The hESC-OPs ${ }^{\mathrm{GFP}+}$ stably expressed high levels of GFP, CD73, CD90, and CD105. They possessed osteogenic differentiation potential in vitro as demonstrated by increased expression of COL1A1, RUNX2, OSTERIX, and OPG transcripts and mineralized nodules positive for Alizarin Red and immunocytochemical expression of osteocalcin, alkaline phosphatase, and collagen-I. In conclusion, we have demonstrated that fluorescently labelled hESC-OPs can maintain their GFP expression for the long term and their potential for osteogenic differentiation in vitro. In future, these fluorescently labelled hESC-OPs could be used for noninvasive assessment of bone regeneration, safety, and therapeutic efficacy.
\end{abstract}

\section{Introduction}

Treatment of posttraumatic or neoplastic bone defects is a major reconstructive challenge. Recent developments in cellular therapy aim to mimic the process of bone repair by delivering cells capable of differentiating into osteoblasts [1]. Sources of such osteoprogenitors in the body include the bone marrow stromal cells (bMSCs) and extraskeletal sources such as adipose-derived stem cells and dental pulp stem cells [2]. Although evidence suggests that bMSCs are the primary source of osteoprogenitor cells [3], their acquisition is complicated by an invasive procedure and inadequate viable cell recovery due to an age-related decline in the number of osteoprogenitor cells [4]. Furthermore, the use of these stem cell sources is limited due to donor availability, healing complications at the donor site, and loss of proliferation capacity upon in vitro expansion [5].

Human embryonic stem cells (hESCs) have recently been proposed as attractive candidate for cellular therapy $[6,7]$. In addition, to its hope for cellular therapies, hESCs also provide a potential in vitro human model to understand early human development and model diseased states and a potential tool for drug screening and toxicology studies [8]. hESCs possess a normal karyotype and maintain high telomerase activity resulting in its indefinite self-renewal potential and possibility for virtually unlimited expansion in vitro [9]. Further, hESCs can differentiate into cell types of all three germ layers $[9,10]$. Hence, hESCs could be utilized to as potentially unlimited source of healthy and functional osteoprogenitors [10]. However, the clinical utility of these stem cells are 
limited by safety and ethical concerns $[6,7]$. Secondly, the limited ability to track the hESC-derived cellular progenies in vivo greatly hinders translational studies. Though evidences suggest the clinical utility of osteoprogenitors-like mesenchymal stem cells in bone regeneration, the exact mechanism is poorly understood. It is proposed that osteoprogenitors could play a role in bone regeneration through homing to the site of injury, cell-cell interactions, and secretion of soluble paracrine factors. Furthermore, lack of reliable markers in vivo restricts the tracking of these cells after transplantation.

Methods such as flow cytometry, immunocytochemistry, and histology have been reliable in characterizing osteogenic differentiation in vitro and in vivo [11]. However, their application is restricted, as they do not allow online monitoring. Fluorescent tags like green fluorescent protein (GFP) allow visualization and real-time monitoring without the need for cellular fixation and immunostaining. Further, they are also valuable tools to control stem cell fate and study cell behaviour during differentiation. Hence, various transgenic hESC lines with constitutive or inducible tissue, cell-specific, or genespecific fluorescent reporters have been generated by various groups [12-16]. Recently, lentiviral-based transduction and targeted gene knock-in methods, like zinc finger nucleases (ZFNs), transcription activator-like effector nucleases (TALENs), and clustered regulatory interspaced short palindromic repeats system (CRISPR/CAS9), are increasingly used as effective tools for gene delivery to hESCs. These methods offer several advantages including high transfection rates, capability for stable transgene expression and/or ability to generate cell-specific promoter-based reporter systems, and targeted genome editing [14-17]. However, some of the major concerns with these systems include insertional mutagenesis, viral integrations, and other off-target effects that can cause genomic instability and disruption of normal gene function [18]. In this regard, nonviral plasmid-based gene delivery methods provide an alternative platform. Nevertheless, in the context of hESCs, nonviral systems are associated with low transfection efficiency which is to certain extent overcome by single cell dissociation of hESCs and small molecule-based methods [19].

In the current study, we assess the ability of fluorescently labelled hESCs (using single cell dissociation and small molecule-mediated nonviral plasmid-based system) to differentiate into osteoprogenitors (hESC-OPs) and investigate their osteogenic differentiation potential.

\section{Materials and Methods}

\subsection{Culture of hESCs}

2.1.1. Feeder-Dependent Culture of hESCs. The NIH-registered H9-hESC cell line, isolated and established at the University of Wisconsin, was used in this study. These cells were cultured on feeder-dependent system using mitomycin$\mathrm{C}$ inactivated murine embryonic fibroblasts (MEFs) and hESC medium as described previously [20]. Briefly, the hESC medium consisted of Dulbecco's modified Eagle's medium (DMEM)/Ham's F12 (1:1) supplemented with 20\% Knockout Serum Replacement (KO-SR; GIBCO), 1\% (vol/vol) nonessential amino acids (Sigma-Aldrich), $1 \mathrm{mM} \mathrm{L-}$ glutamine (GIBCO), $4 \mathrm{ng} / \mathrm{mL}$ basic fibroblast growth factor (bFGF; Invitrogen), and $0.1 \mathrm{mM} \beta$-mercaptoethanol (SigmaAldrich). Media were changed every other day and passaged every 6-7 days using $1 \mathrm{mg} / \mathrm{mL}$ collagenase type IV (GIBCO) for 5 minutes, followed by manual dissociation to small clumps and seeding onto MEF-seeded plates.

2.1.2. Fluorescent Labelling of hESCs. H9-hESC colonies were dissociated into single cells using 5-minute incubation with Accutase (StemCell Technologies), suspended in hESC medium supplemented with Y-27632 (inhibitor of Rhoassociated protein kinase, ROCK, $10 \mu \mathrm{M}$, Stemgent), and seeded onto MEF-seeded six-well plates. Plasmid construct consisted of pAcGFP1-1 backbone with pCAG-GFP promoter (Clonetech). hESCs were transfected with GFP plasmid using $\mathrm{X}$-tremeGENE HP lipid-based transfection reagent (Roche) according to manufacturer's instructions. Briefly, $2 \mu \mathrm{g}$ of plasmid DNA and $6 \mu \mathrm{L}$ of X-tremeGENE HP transfection reagent in $200 \mu \mathrm{L}$ of hESC medium were incubated for 15 minutes at room temperature and added drop-by-drop to freshly seeded hESCs. Cells were cultured normally with daily change of media. 72 hours after transfection, GFP positive hESCs were selectively picked and transferred to new wells for expansion. Selection process was repeated until relatively homogenous GFP positive hESCs colonies were established over the next 5 passages. These hESCs stably expressed GFP for over 30 passages and are referred to as H9-hESC ${ }^{\mathrm{GFP}+}$.

2.1.3. Feeder-Free Culture of Fluorescently Labelled hESCs. To establish a feeder-free system, H9-hESCs ${ }^{\mathrm{GFP}+}$ were transitioned and subcultured over Matrigel ${ }^{\mathrm{TM}}$ (BD Biosciences) in complete $\mathrm{mTeSR}^{\mathrm{TM}} 1$ medium (StemCell Technologies). Under these conditions, the hESCs maintained their undifferentiated state and also stably expressed GFP. Confluent feederfree hESC cultures were passaged every 5-6 days using $1 \mathrm{mg} / \mathrm{mL}$ Dispase (StemCell Technologies) for 5 minutes, followed by manual dissociation to small clumps and replating onto freshly prepared Matrigel coated plates as previously described [21].

\subsection{Differentiation of Fluorescently Labelled hESCs to hESC-OPs}

\subsubsection{Phase 1: Spin-EB Formation Using Forced Aggregation.} H9-hESC ${ }^{\mathrm{GFP}+}$ colonies were dissociated into single cells using 10-15-minute incubation with Accutase. Predetermined numbers of single cell dissociated hESCs $\left(6 \times 10^{6}\right.$ cells) were seeded onto each well of Aggrewell $^{\mathrm{TM}} 800$ plates (StemCell Technologies) in Aggrewell media (StemCell Technologies) supplemented with Y27632 $(10 \mu \mathrm{M})$. The single cell dissociated cells were forcibly aggregated onto the microwells by centrifugation at $100 \times \mathrm{g}$ for 3 minutes. The single cell dissociated hESCs formed clusters of uniform size within the microwells after overnight incubation. These clusters of hESCs would be referred to as spin-embryoid bodies (spinEBs). The spin-EBs were harvested after 24 hours and transferred to ultralow-attachment six-well culture plates (Corning) in Aggrewell media for 5 days. 
2.2.2. Phase 2: Differentiation of Spin-EBs into Osteoprogenitors. After 5 days of differentiation, spin-EBs were collected and plated onto $1 \mu \mathrm{g} / \mathrm{cm}^{2}$ fibronectin (GIBCO) coated tissue culture plates in Aggrewell medium until confluence (approximately 15-20 days). After confluence, the spin-EB outgrowths were trypsinized and cultured in Mesenchymal Stem Cell Growth Medium (PromoCell) for 3-8 passages. The EB outgrowths attained homogenous population of spindleshaped cells from 3rd passage. These spindle-shaped cells were characterized for osteoprogenitor-related markers and would be referred to as $\mathrm{H} 9-\mathrm{hESCs}{ }^{\mathrm{GFP}+}$ derived osteoprogenitors (hESC-OPs ${ }^{\mathrm{GFP}+}$ ).

2.3. RNA Extraction and Real-Time Reverse TranscriptasePolymerase Chain Reaction ( $q R T-P C R)$. Total RNA was extracted using RNeasy Plus Mini Kit (Qiagen) according to the manufacturer's protocol. Then, $500 \mathrm{ng}$ of total RNA was used to generate cDNA using iScript cDNA synthesis kit (BioRad) and the cDNA was used as template for qRT-PCR. Relative expression levels of respective genes were analyzed using StepOne Plus ${ }^{\mathrm{TM}}$ real-time thermocycler (Applied Biosystems) and Fast SYBR Green PCR Master Mix System (Applied Biosystems). For relative quantification, the expression levels of respective genes were normalized to that of $\beta$-Actin and expressed as a fold change relative to the expression levels in undifferentiated hESCs.

2.4. Flow Cytometry Analysis. The differentiated hESCs (hESC-OPs ${ }^{\mathrm{GFP}+}$ ) were dissociated into single cells using Accutase (StemCell Technologies) for 5 minutes at $37^{\circ} \mathrm{C}$, resuspended in flow cytometry buffer (PBS containing $0.5 \%$ bovine serum albumin), and passed through $40 \mu \mathrm{m}$ cell strainer. Nonspecific binding of the antibodies was inhibited by incubating the cells with $\mathrm{FcR} \gamma$ blocking agent (Miltenyi Biotec) for $10 \mathrm{mins}$ at $4^{\circ} \mathrm{C}$. For labelling of cell surface antigens, the cells were incubated with antibodies against CD73APC (eBioscience), CD90-PE (BD Pharmingen), CD105-PE (BD Pharmingen), and CD45-PerCP (BD Pharmingen) at $4^{\circ} \mathrm{C}$ for 10 minutes. After washing with flow cytometry buffer, the antibody-labelled cells were analyzed using DakoCytomation Cyan ADP and Summit v4.3 software.

\subsection{Osteogenic Differentiation of hESC-OPs ${ }^{G F P+}$. hESC-} $\mathrm{OPs}^{\mathrm{GFP}+}$ were differentiated towards osteogenic lineage using MSC osteogenic differentiation medium (PromoCell). Briefly, hESC-OPs ${ }^{\mathrm{GFP}+}$ were plated in a tissue culture plates (seeding density: $3 \times 10^{4}$ cells $/ \mathrm{cm}^{2}$ ) using MSC growth medium. When the cells were almost $100 \%$ confluent (1-2 days), osteogenic differentiation was induced with MSC osteogenic differentiation medium (PromoCell) as per manufacturer's instructions. MSC growth medium was used as a negative control. The cells were incubated for 14-28 days, with media changed every third day.

2.6. Alizarin Red Staining. Alizarin Red staining was used to identify calcific nodules produced by cells after 28 days of osteogenic differentiation. Cultures were washed with PBS and fixed with $4 \%$ paraformaldehyde for 20 minutes at room temperature. Cultures were then washed and incubated with Alizarin Red for one minute, washed thoroughly, and air-dried. Formation of chromogenic complex between o-cresolphthalein and calcium ions was visualized using inverted light microscope (Olympus IX70).

2.7. Immunocytochemical Staining. hESC-OPs ${ }^{\mathrm{GFP}+}$ cells differentiated under osteogenic conditions for 14 days were washed with PBS and fixed in 4\% paraformaldehyde for 20 minutes at room temperature. For detection of intracellular proteins, the cells were permeabilized with $0.1 \%$ Triton X100 (Sigma-Aldrich) in PBS and blocked with 10\% goat serum $/ 2 \%$ BSA in PBS for 1 hour at room temperature. Then the cells were incubated with primary antibodies against mouse anti-human GFP (1:200, Santa Cruz Biotech), rabbit anti-human collagen-1 (1:300, Millipore), rabbit anti-human osteocalcin (1:200, AbD Serotec), and rabbit anti-human alkaline phosphatase $(1: 200$, Santa Cruz Biotech) in blocking solution at $4^{\circ} \mathrm{C}$ overnight. Then, the cultures were washed thrice in PBST (0.1\% Tween-20 in PBS) and incubated with goat anti-mouse Alexa Fluor-488 and goat anti-rabbit Alexa Flour-594 conjugated secondary antibodies (1:200, Molecular Probes) for 1 hour at room temperature, followed by nuclear labelling with $4^{\prime}, 6$-diamidino-2-phenylindole dihydrochloride (DAPI) for 5 minutes at room temperature. The cultures were then washed thrice with PBS. Immunostained cultures were observed and imaged using Olympus IX70 fluorescence microscope.

2.8. Statistical Analysis. The results of qRT-PCR are presented as mean \pm standard deviation of three experiments. Statistical differences were evaluated by a two-tailed Student's $t$-test. $p$ values $<0.05$ were considered as statistically significant.

\section{Results}

3.1. Differentiation of Fluorescently Labelled hESCs into hESC$O P s$. To generate fluorescently labelled osteoprogenitor-like cells from hESCs, H9-hESCs were first fluorescently labelled with GFP and adapted to feeder-free culture system (Figure $1(\mathrm{a})$ ). These H9-hESCs ${ }^{\mathrm{GFP}+}$ were differentiated through a two-stage EB-outgrowth method under serum-free conditions similar to a method previously described for differentiation of hESCs to MSC-like phenotype [10, 22]. Briefly, spin-EBs were generated by forced aggregation of single cell dissociated hESC colonies followed by suspension culture of hESC aggregates under ultralow-attachment and serumfree conditions (Figure 1(b)). After 5 days of differentiation under suspension culture, spin-EBs were plated onto fibronectin-coated plates for outgrowth of differentiated cells (Figure 1(c)). Immediately after adherent culture of spin-EBs, cells with different morphologies varying from epithelioid to spindle-shape migrated out from the spin-EBs. Upon serial subculture, spindle-shaped cells outnumbered other cell types and attained homogeneous morphology after 3 passages (Figure 1(d)). The hESC-derived cells stably expressed GFP throughout the differentiation process resulting in the differentiation of fluorescently labelled hESCs $\left(\mathrm{H} 9-\mathrm{hESCs}^{\mathrm{GFP}+}\right)$ to osteoprogenitor-like cells (hESC-OPs ${ }^{\mathrm{GFP}+}$ ) (Figure 1). 

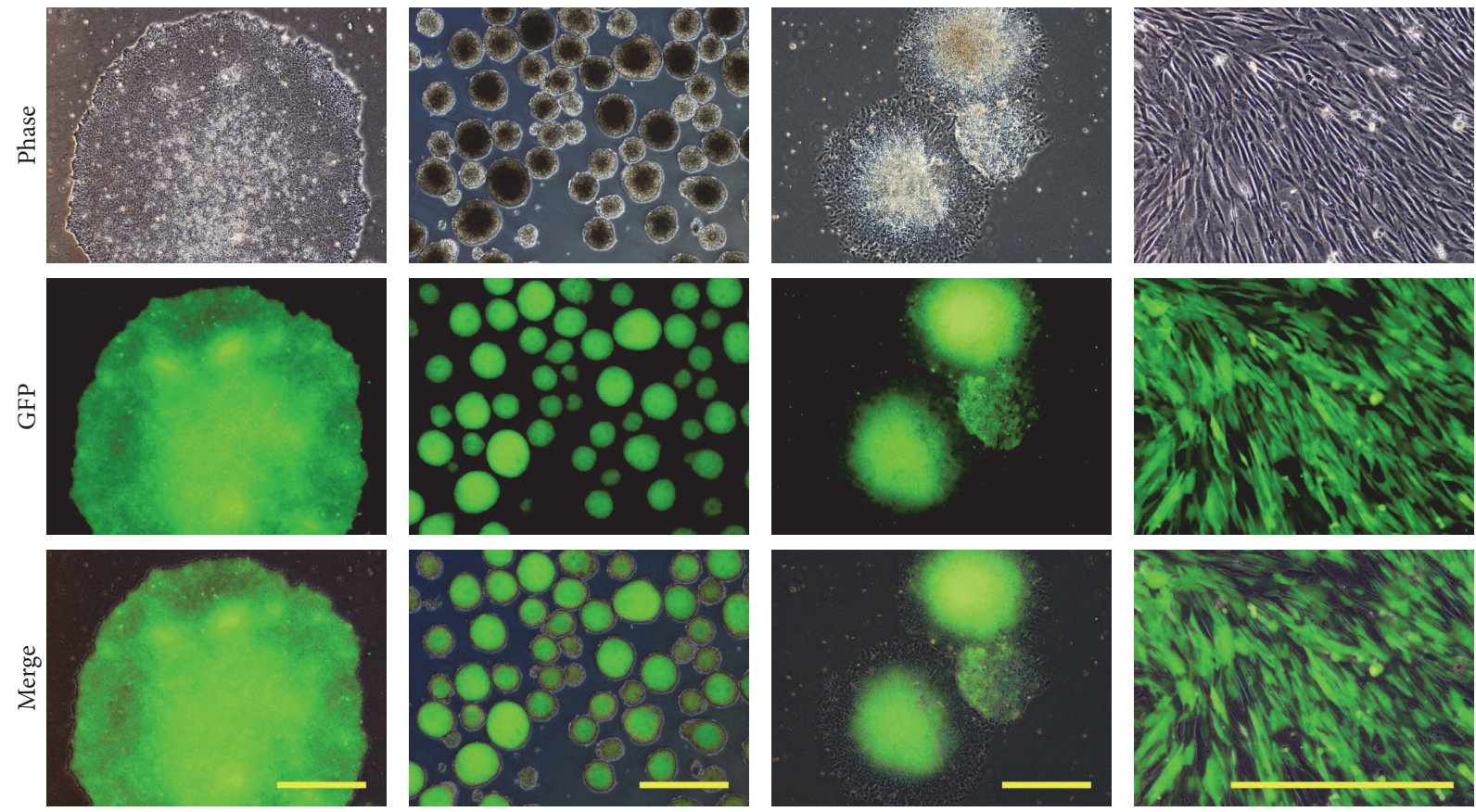

hESCs

(GFP+)

(a)
Embryoid bodies

(b)
EB-outgrowth

(c)
hESC-OPs

(d)

FIGURE 1: Differentiation of GFP-expressing hESCs into hESC-OPs. The phase contrast, fluorescent, and merged photomicrographs demonstrate the differentiation of GFP-expressing hESCs (a) into embryoid bodies (b), embryoid body outgrowth, (c) and hESC-OPs (d). Scale bar: $500 \mu \mathrm{m}$.

3.2. Characterization of Fluorescently Labelled hESC-OPs. The phenotype of fluorescently labelled hESC-OPs ${ }^{\mathrm{GFP}+}$ was characterized using qRT-PCR and flow cytometry. qRT-PCR analysis of hESC-OPs ${ }^{\mathrm{GFP}+}$ demonstrates the downregulation of transcripts related to pluripotency (OCT4, SOX2, and $N A N O G$ ) and chondrogenic (COL2A1) lineage accompanied by upregulation of mesenchymal (COL1A1 and COL3A1) and osteogenic (OSTERIX, RUNX2, and OPG) lineage-associated transcripts (Figure 2).

The immunophenotype of hESC-OPs ${ }^{\mathrm{GFP}+}$ was analyzed using flow cytometry for surface markers. The hESC-OPs GFP+ demonstrated strong expression of GFP (96.6\%) and mesenchymal stem cell-associated surface markers CD73 (99.1\%), CD90 (97.6\%), and CD105 (96.5\%). These cells were negative for hematopoietic marker CD45 $(<1 \%)$ (Figure 3).

3.3. Characterization of Osteogenic Differentiation of Fluorescently Labelled hESC-OPs. The osteogenic differentiation ability of hESC-OPs ${ }^{\mathrm{GPP}+}$ was characterized using qRTPCR, Alizarin Red staining, and immunocytochemistry. qRT-PCR analysis demonstrates the significant upregulation of transcripts (COL1A1, RUNX2, and OSTERIX) associated with osteogenic differentiation except OPG (Figure 4). Under osteogenic conditions, hESC-OPs ${ }^{\mathrm{GFP}+}$ formed large mineralized nodules that stained intensely red with Alizarin Red (Figure 5). Immunocytochemical staining of hESC-OPs ${ }^{\text {GFP+ }}$ differentiated under osteogenic conditions for 14 days demonstrates the strong expression of osteocalcin, alkaline phosphatase, and collagen-I (Figure 6).

Overall, these results suggest that the efficient differentiation of fluorescently labelled hESCs into hESC-OPs stably expressed GFP and demonstrated osteogenic differentiation in vitro.

\section{Discussion}

Poor survival of transplanted cells and safety concerns related to formation of ectopic tissues and tumors raise the crucial need for establishing an effective technique for tracing the hESCs and their differentiated progenies in vivo for tissue engineering related applications. Fluorescent dyes, such as DiL, Hoechst 33342, CFSE (carboxyfluorescein succinimidyl ester), and PKH linker dyes, have been used to trace the presence and activity of transplanted cells [23-25]. However, these cytoplasmic or nuclear markers lose their intensity as the cells proliferate. In addition, these dyes could be taken up nonspecifically by the host cells in vivo and hence impair long-term tracing studies and may provide misleading information on donor survival and differentiation $[23,26]$.

The incorporation of GFP into the genome of hESCs that is stably expressed in the differentiated progenies provides a proof-of-concept to trace their presence, migration, and formation of tissues after transplantation [27]. An elegant study proved the existence of bipotent hemangioblasts that are capable of differentiating into endothelial and hematopoietic 

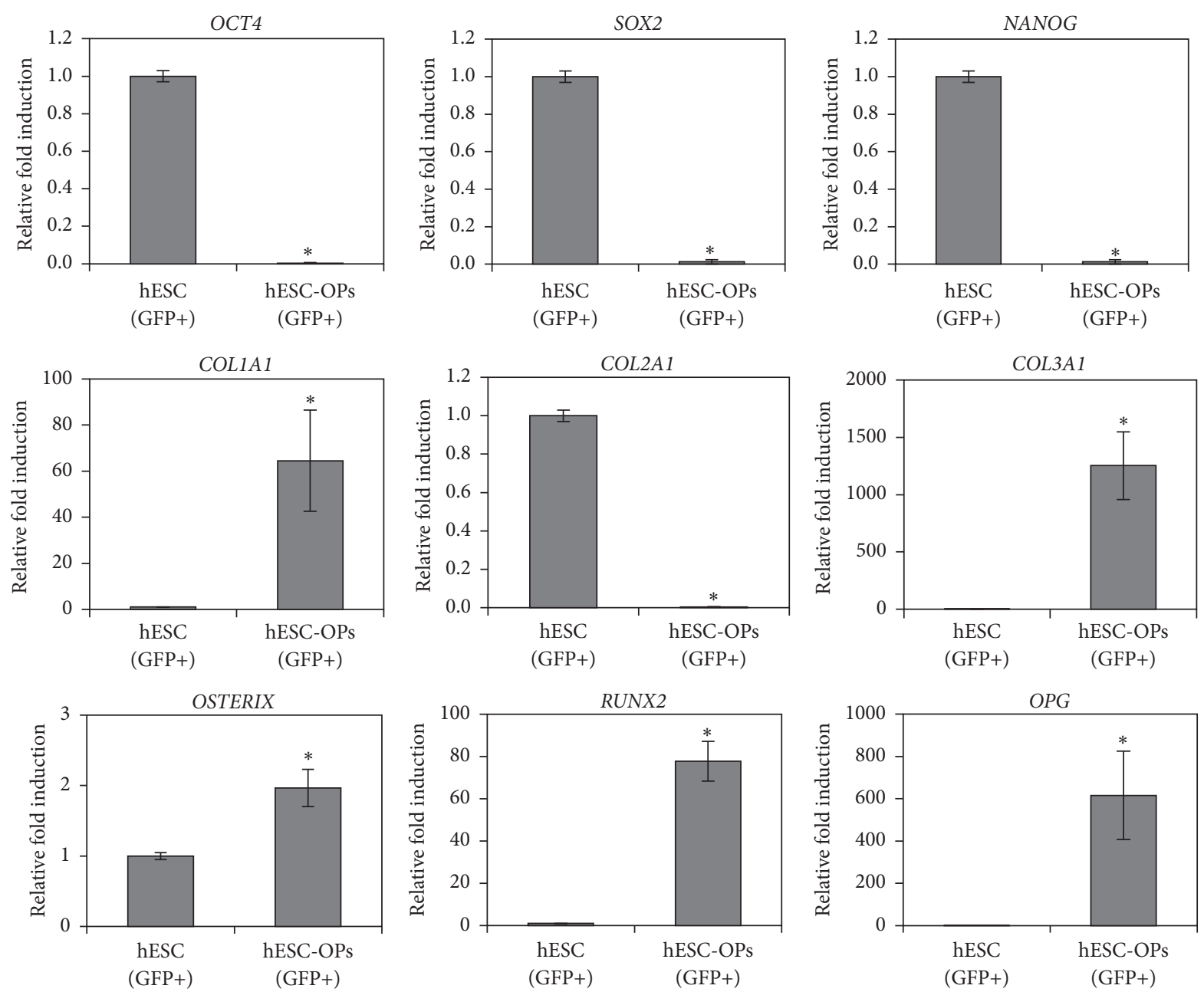

FIGURE 2: Characterization of hESC-OPs (GFP+) by real-time RT-PCR for pluripotency (OCT4, SOX2, and NANOG), mesenchymal (COL1A1 and COL3A1), chondrogenic (COL2A1), and osteogenic (RUNX2, OSTERIX, and OPG) lineage-associated transcripts. The transcript levels were normalized to respective $\beta$-Actin levels and to hESC (GFP+) cells. Values represent the means \pm SD of three experiments $\left({ }^{*} p<0.05\right.$ versus hESC control).
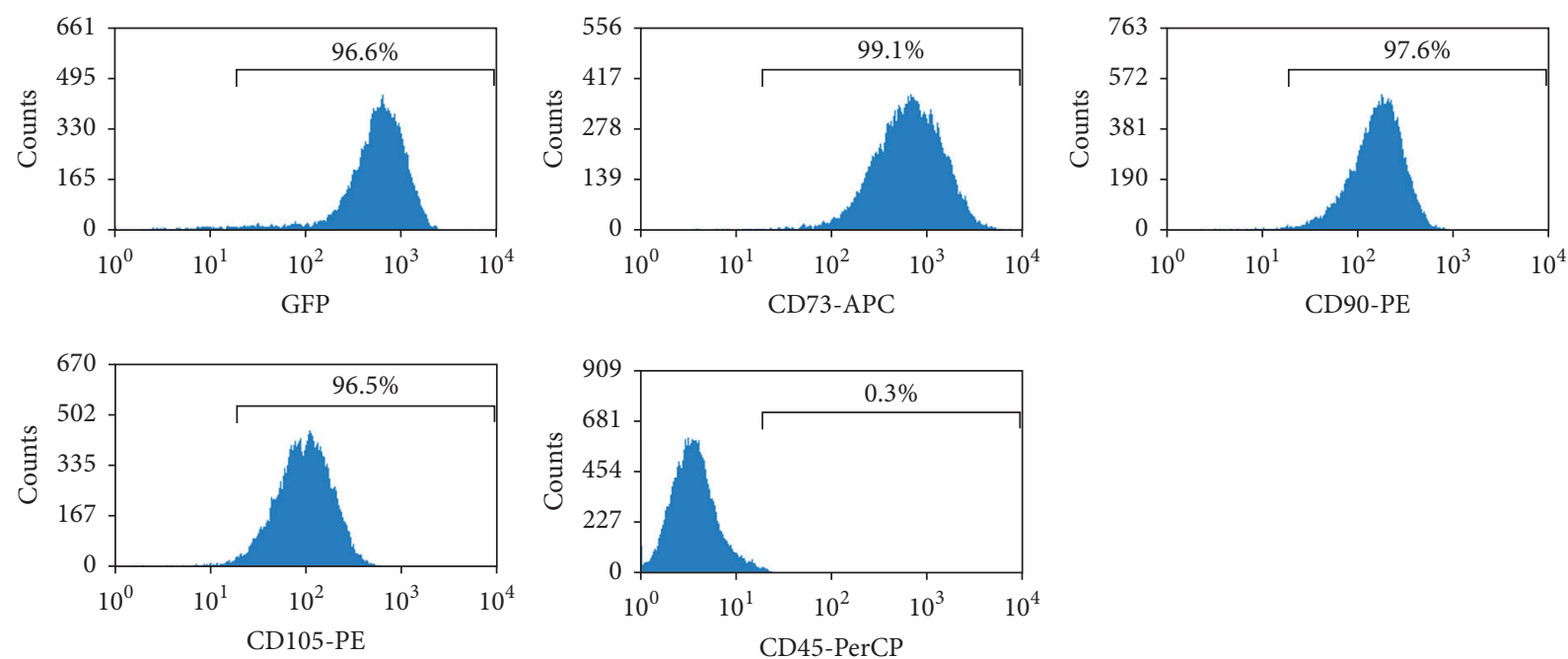

FIgURE 3: Immunocharacterization of hESC-OPs (GFP+) using flow cytometry for expression of surface markers. 

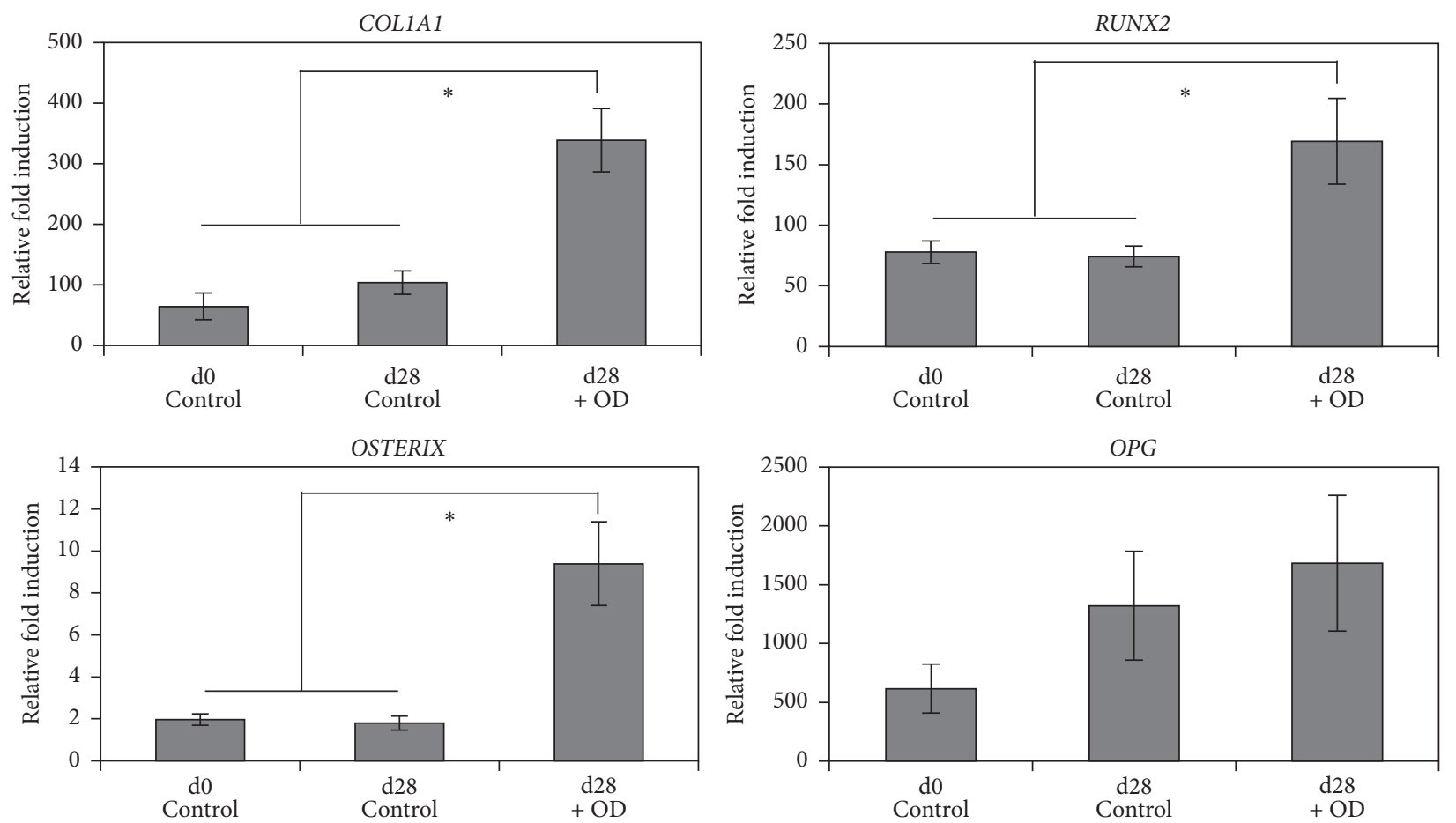

FIgURE 4: Characterization of osteogenic differentiation of hESC-OPs (GFP+) by real-time RT-PCR. The transcript levels were normalized to respective $\beta$-Actin levels and to hESC (GFP+) cells. Values represent the means \pm SD of three experiments $\left({ }^{*} p<0.05\right.$ versus respective controls). hESC-OPs (GFP+) were differentiated for 4 weeks in the presence of osteogenic factors (OD). Day 0 hESC-OPs (GFP+) and cells differentiated for 4 weeks without osteogenic factors were used as controls.
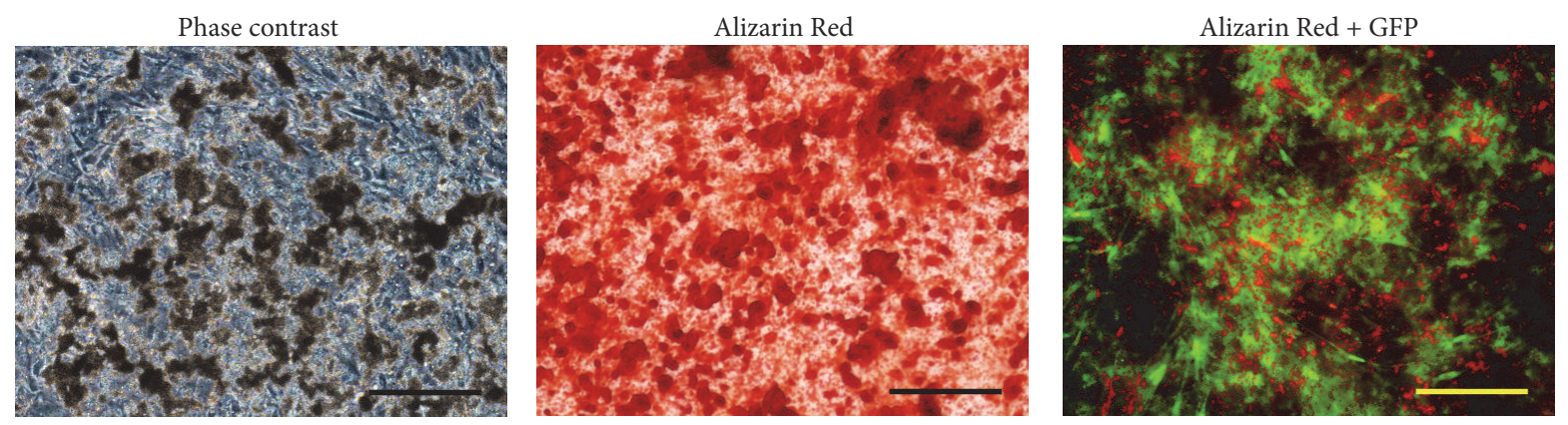

FIGURE 5: Osteogenic differentiation of GFP-expressing hESC-OPs demonstrated using Alizarin Red staining. Scale bar: $200 \mu \mathrm{m}$.

cell lineages using GFP-labelled hESCs-derived blast colonies transplanted into various mouse ischemia models [28]. Similarly, other studies have shown the utilization of fluorescently labelled hESC-derived endothelial cells to trace and evaluate the formation and integration of functional blood vessels in vivo $[29,30]$ and their transition to hematopoietic cells [31].

Due to the genomic integration of GFP, it is important to ensure the continued proliferation and differentiation ability of the stem cell population. Previous studies have shown successful transduction using lentiviral vectors encoding fluorescent proteins in human adipose-derived stem cells with potential for adipogenic and osteogenic differentiation [32] and in human placenta-derived MSCs with potential for adipogenic, osteogenic, and hepatic differentiation [33]. In a recent study, Ovchinnikov et al. used a lentiviral-based delivery system to generated transgenic pluripotent stem cells (PSC) lines that expressed pluripotency-driven GFP [13]. These cell lines effectively report for pluripotency and hence play a role in detection of PSCs from their differentiated progenies. Similarly, Zou et al. generated a RUNX2 promoter driven YFP based reporter system in hESCs to track their commitment to osteogenic lineage [15]. Using these transgenic hESCs, they elegantly demonstrated that BMP2 does not induce osteogenic differentiation but promotes the generation of CD73+ osteoprogenitors and their osteogenic differentiation. In a recent study, Zhou et al. generated choline acetyl transferase (ChAT) promoter driven zsGreen expressing hESC line that labels cholinergic neuronal differentiation [14]. Overall, these reporter hESC lines not only provide an opportunity to monitor lineage specific differentiation but 

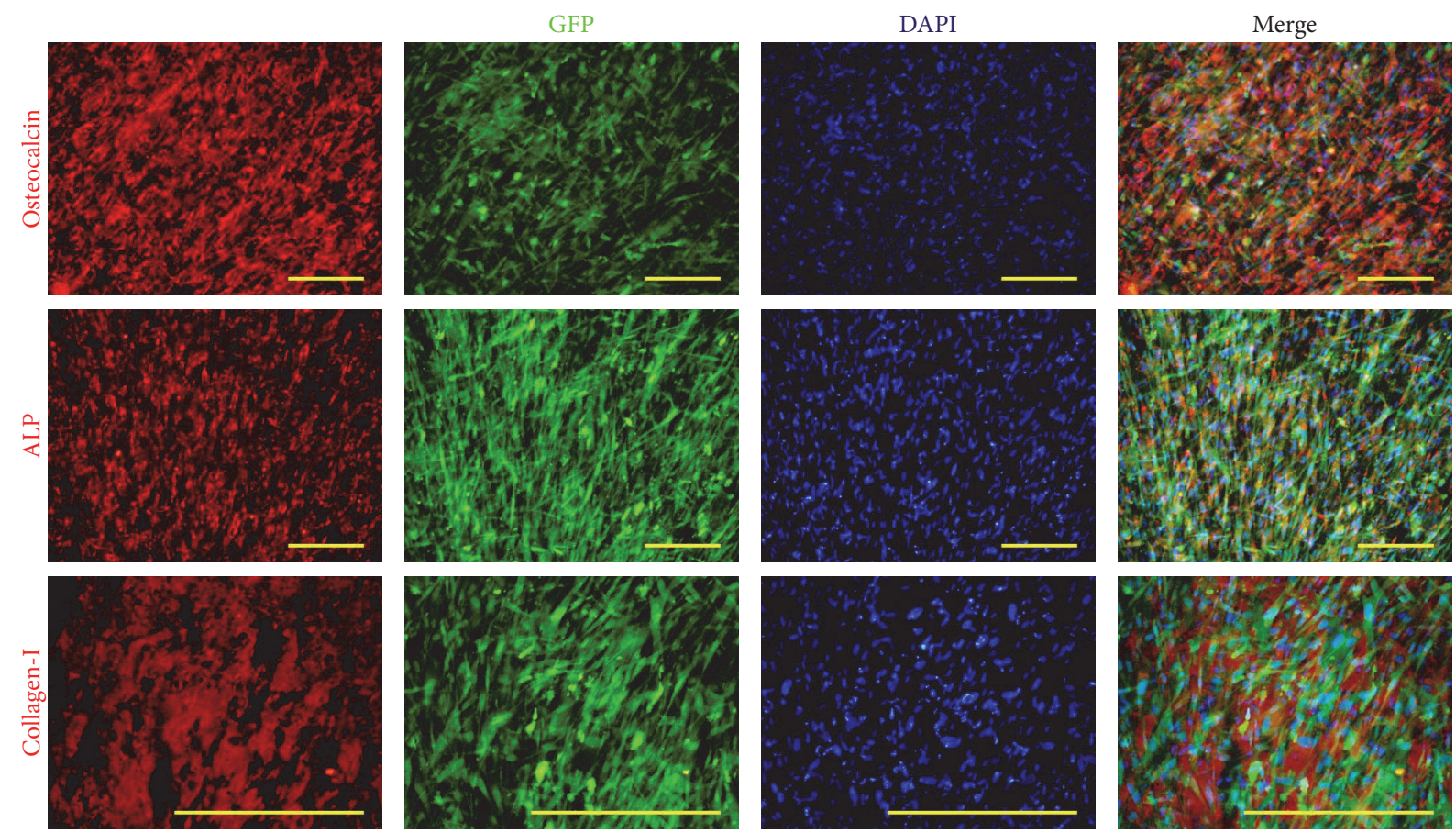

FIGURE 6: Osteogenic differentiation of GFP-expressing hESC-OPs demonstrated using immunocytochemical staining for osteocalcin, alkaline phosphatase (ALP), and collagen-I after 14 days of induction in osteogenic differentiation conditions. Scale bar: $500 \mu \mathrm{m}$.

also aid in study of physiological and biochemical pathways controlling the differentiation. In this study, we have shown the stable expression of GFP in hESCs and their differentiated progeny (hESC-OPs). The expression of surface markers (CD73, CD90, and CD105), data from gene expression analysis, and Alizarin Red and immunofluorescent staining demonstrate the osteogenic commitment and differentiation potential of these GFP-expressing hESCs. These results are in agreement with previous studies [10, 15, 20, 34, 35]. These data also suggest the stable expression of GFP in hESC-OPs and the genetic manipulation did not affect their osteogenic differentiation.

An elegant study demonstrated the comparative efficiency and effect of different viral and nonviral vectors on rat MSCs and their differentiation potential [36]. This study demonstrated superior efficacy of gene transfer and lower toxicity using lentiviral-based methods compared to adenoviral based methods. Further, transfection using plasmid DNA is moderately effective compared to the high efficiency of transduction using lentiviral-based methods of gene transfer. Similarly, another study using human MSCs has demonstrated the higher efficiency of lentiviral-based transduction of GFP compared to oncoretroviral vectors [37]. However, the lentiviral GFP transduction seemed to impair in vitro osteogenesis to a certain extent, which might be due to lentiviral factors or GFP gene itself. It is also known that GFP can be toxic to certain cell types resulting in lack of protein expression [38] and phenotypic changes [39] or have subtle effects on differentiation [37]. Conventionally, nonviral plasmid DNA vectors are less efficient in gene transfers but are advantageous due to their lower toxicity. In the context of hESCs, this is important as hESCs upon dissociation into single cells are extremely prone for cell death and apoptosis. Recently, Yen et al. developed a nonviral, plasmid-based fluorescently labelling of hESCs with moderately high transfection efficiencies through single cell dissociation of hESCs and use of small molecules during the exposure to plasmid [19]. In this study, we used a similar approach to transfect the hESCs. However, the efficiency of the transfection were not quantified as the selection process was carried out through manual selection over several passages.

The ability of fluorescently labelled hESCs to differentiate to hESC-OPs and further to osteogenic lineage provides a platform to trace the osteogenic potential in tissue engineering related applications. However, additional studies are necessary to determine the engraftment and in vivo bone regeneration potential of these fluorescently labelled hESCOPs. However, we do not propose to utilize the fluorescently labelled hESC progenies for human clinical translational applications. The use of GFP-labelled hESC-OPs would be extremely useful in noninvasive imaging of bone regeneration within three-dimensional (3D) in vitro scaffolds and in vivo animal models. Recently, several studies have produced bone-like matrix by seeding human PSCs on different 3D scaffolds [40-43] and using perfusion bioreactors [44, 45]. Jeon et al. used a biomimetic approach to regenerate bone by $3 \mathrm{D}$ coculture of human PSC-derived osteoblasts and osteoclasts within a synthetic scaffolds [46]. We have recently developed protocols to direct the differentiation of hESCs to vascular cells [20,47]. In future studies, we plan to address the 
osteodifferentiation potential of hESC-OPs cocultured with vascular cells within 3D scaffolds to generate vascularized bone tissues.

\section{Conclusion}

In this study, we have demonstrated that hESCs fluorescently labelled using plasmid vectors encoding GFP can be efficiently differentiated into hESC-OPs. These hESC-OPs maintain their GFP expression for the long term and their potential for osteogenic differentiation in vitro. Plasmidbased GFP transfection provides a direct and simple method to label hESCs and their progenies and does not impede their in vitro differentiation potential. This technique provides opportunities to investigate bone regeneration using noninvasive imaging modalities. However, extensive longterm in vivo studies are required to access the safety, efficacy, and therapeutic potential of these cells.

\section{Competing Interests}

The authors declare that they have no competing interests.

\section{Acknowledgments}

This work was partially supported by the National University Health System Research Grant R-223-000-024-133 and National University Health System B2B Grant R-221000-074-515, Singapore. Gopu Sriram, Mingming Li, Yu Zou, and Harish Handral were supported by NUS Research Scholarship. Gopu Sriram was also supported by President Graduate Fellowship.

\section{References}

[1] T. J. Voegele, M. Voegele-Kadletz, V. Esposito et al., "The effect of different isolation techniques on human osteoblast-like cell growth," Anticancer Research, vol. 20, no. 5, pp. 3575-3582, 2000.

[2] P. Bosch, D. S. Musgrave, J. Y. Lee et al., "Osteoprogenitor cells within skeletal muscle," Journal of Orthopaedic Research, vol. 18, no. 6, pp. 933-944, 2000.

[3] S. P. Bruder, N. Jaiswal, N. S. Ricalton, J. D. Mosca, K. H. Kraus, and S. Kadiyala, "Mesenchymal stem cells in osteobiology and applied bone regeneration," Clinical Orthopaedics and Related Research, no. 355, pp. S247-S256, 1998.

[4] S. Nishida, N. Endo, H. Yamagiwa, T. Tanizawa, and H. E. Takahashi, "Number of osteoprogenitor cells in human bone marrow markedly decreases after skeletal maturation," Journal of Bone and Mineral Metabolism, vol. 17, no. 3, pp. 171-177, 1999.

[5] R. Siddappa, R. Licht, C. van Blitterswijk, and J. de Boer, "Donor variation and loss of multipotency during in vitro expansion of human mesenchymal stem cells for bone tissue engineering," Journal of Orthopaedic Research, vol. 25, no. 8, pp. 1029-1041, 2007.

[6] C. Unger, H. Skottman, P. Blomberg, M. Sirac dilber, and O. Hovatta, "Good manufacturing practice and clinical-grade human embryonic stem cell lines," Human Molecular Genetics, vol. 17, no. 1, pp. R48-R53, 2008.

[7] M. X. Doss, C. I. Koehler, C. Gissel, J. Hescheler, and A. Sachinidis, "Embryonic stem cells: a promising tool for cell replacement therapy," Journal of Cellular and Molecular Medicine, vol. 8, no. 4, pp. 465-473, 2004.

[8] P. Menendez, C. Bueno, and L. Wang, "Human embryonic stem cells: a journey beyond cell replacement therapies," Cytotherapy, vol. 8, no. 6, pp. 530-541, 2006.

[9] J. S. Odorico, D. S. Kaufman, and J. A. Thomson, "Multilineage differentiation from human embryonic stem cell lines," Stem Cells, vol. 19, no. 3, pp. 193-204, 2001.

[10] X. Fu, Y. Chen, F.-N. Xie et al., "Comparison of immunological characteristics of mesenchymal stem cells derived from human embryonic stem cells and bone marrow," Tissue Engineering Part A, vol. 21, no. 3-4, pp. 616-626, 2015.

[11] M.-L. Yen, C.-C. Chien, I.-M. Chiu et al., "Multilineage differentiation and characterization of the human fetal osteoblastic 1.19 cell line: a possible in vitro model of human mesenchymal progenitors," Stem Cells, vol. 25, no. 1, pp. 125-131, 2007.

[12] R. Shetty, D. Shetty, and M. S. Inamdar, "Generation of human embryonic stem cell line expressing green fluorescent protein," Stem Cell Research, vol. 16, no. 2, pp. 311-313, 2016.

[13] D. A. Ovchinnikov, D. M. Titmarsh, P. R. J. Fortuna et al., "Transgenic human ES and iPS reporter cell lines for identification and selection of pluripotent stem cells in vitro," Stem Cell Research, vol. 13, no. 2, pp. 251-261, 2014.

[14] J. Zhou, C. Wang, K. Zhang et al., "Generation of human embryonic stem cell line expressing zsgreen in cholinergic neurons using CRISPR/Cas9 system," Neurochemical Research, vol. 41, no. 8, pp. 2065-2074, 2016.

[15] L. Zou, F. K. Kidwai, R. A. Kopher et al., "Use of RUNX2 expression to identify osteogenic progenitor cells derived from human embryonic stem cells," Stem Cell Reports, vol. 4, no. 2, pp. 190-198, 2015.

[16] Y. Liu, P. Jiang, and W. Deng, "OLIG gene targeting in human pluripotent stem cells for motor neuron and oligodendrocyte differentiation," Nature Protocols, vol. 6, no. 5, pp. 640-655, 2011.

[17] S. C. Den Hartogh and R. Passier, "Concise review: fluorescent reporters in human pluripotent stem cells: contributions to cardiac differentiation and their applications in cardiac disease and toxicity," Stem Cells, vol. 34, no. 1, pp. 13-26, 2016.

[18] Y. Fu, J. A. Foden, C. Khayter et al., "High-frequency off-target mutagenesis induced by CRISPR-Cas nucleases in human cells," Nature Biotechnology, vol. 31, no. 9, pp. 822-826, 2013.

[19] J. Yen, L. Yin, and J. Cheng, "Enhanced non-viral gene delivery to human embryonic stem cells via small molecule-mediated transient alteration of the cell structure," Journal of Materials Chemistry B, vol. 2, no. 46, pp. 8098-8105, 2014.

[20] G. Sriram, V. P. Natu, I. Islam et al., "Innate immune response of human embryonic stem cell-derived fibroblasts and mesenchymal stem cells to periodontopathogens," Stem Cells International, vol. 2016, Article ID 8905365, 15 pages, 2016.

[21] G. Sriram, J. Y. Tan, I. Islam, A. J. Rufaihah, and T. Cao, "Efficient differentiation of human embryonic stem cells to arterial and venous endothelial cells under feeder- and serumfree conditions," Stem Cell Research and Therapy, vol. 6, no. 1, article 261, 2015.

[22] F. K. Kidwai, M. M. Movahednia, K. Iqbal, D. S. Jokhun, T. Cao, and A. S. Fawzy, "Human embryonic stem cell differentiation into odontoblastic lineage: an in vitro study," International Endodontic Journal, vol. 47, no. 4, pp. 346-355, 2014.

[23] N. Mohorko, N. Kregar-Velikonja, G. Repovš, M. Gorensek, and M. Bresjanac, "An in vitro study of Hoechst 33342 redistribution and its effects on cell viability," Human and Experimental Toxicology, vol. 24, no. 11, pp. 573-580, 2005. 
[24] B. Rieck and S. Schlaak, "In vivo tracking of rat preadipocytes after autologous transplantation," Annals of Plastic Surgery, vol. 51, no. 3, pp. 294-300, 2003.

[25] L. L. Wang, Y. Y. Qi, Y. Z. Jiang et al., Human Embryonic Stem Cell-Derived Mesenchymal Stem Cells and BMP7 Promote Cartilage Repair, Springer, Berlin, Germany, 2009.

[26] T. M. Coyne, A. J. Marcus, D. Woodbury, and I. B. Black, "Marrow stromal cells transplanted to the adult brain are rejected by an inflammatory response and transfer donor labels to host neurons and glia," STEM CELLS, vol. 24, no. 11, pp. 24832492, 2006.

[27] S. Wolbank, A. Peterbauer, E. Wassermann et al., "Labelling of human adipose-derived stem cells for non-invasive in vivo cell tracking," Cell and Tissue Banking, vol. 8, no. 3, pp. 163-177, 2007.

[28] S.-J. Lu, Q. Feng, S. Caballero et al., "Generation of functional hemangioblasts from human embryonic stem cells," Nature Methods, vol. 4, no. 6, pp. 501-509, 2007.

[29] Z. Z. Wang, P. Au, T. Chen et al., "Endothelial cells derived from human embryonic stem cells form durable blood vessels in vivo," Nature Biotechnology, vol. 25, no. 3, pp. 317-318, 2007.

[30] D. James, H.-S. Nam, M. Seandel et al., "Expansion and maintenance of human embryonic stem cell-derived endothelial cells by TGFbeta inhibition is Id1 dependent," Nature Biotechnology, vol. 28, no. 2, pp. 161-166, 2010.

[31] S. Rafii, C. C. Kloss, J. M. Butler et al., "Human ESC-derived hemogenic endothelial cells undergo distinct waves of endothelial to hematopoietic transition," Blood, vol. 121, no. 5, pp. 770780, 2013.

[32] Q. Wang, M. B. Steigelman, J. A. Walker et al., "In vitro osteogenic differentiation of adipose stem cells after lentiviral transduction with green fluorescent protein," The Journal of Craniofacial Surgery, vol. 20, no. 6, pp. 2193-2199, 2009.

[33] J. Yu, X. Su, C. Zhu et al., "GFP labeling and hepatic differentiation potential of human placenta-derived mesenchymal stem cells," Cellular Physiology and Biochemistry, vol. 35, no. 6, pp. 2299-2308, 2015.

[34] L. Harkness, A. Mahmood, N. Ditzel, B. M. Abdallah, J. V. Nygaard, and M. Kassem, "Selective isolation and differentiation of a stromal population of human embryonic stem cells with osteogenic potential," Bone, vol. 48, no. 2, pp. 231-241, 2011.

[35] L. T. Kuhn, Y. Liu, N. L. Boyd et al., "Developmental-like bone regeneration by human embryonic stem cell-derived mesenchymal cells," Tissue Engineering-Part A, vol. 20, no. 1-2, pp. 365-377, 2014.

[36] J. M. Mcmahon, S. Conroy, M. Lyons et al., "Gene transfer into rat mesenchymal stem cells: a comparative study of viral and nonviral vectors," Stem Cells and Development, vol. 15, no. 1, pp. 87-96, 2006.

[37] A. N. Van Damme, L. Thorrez, L. Ma et al., "Efficient lentiviral transduction and improved engraftment of human bone marrow mesenchymal cells," STEM CELLS, vol. 24 , no. 4, pp. 896907, 2006.

[38] Y. Hanazono, J.-M. Yu, C. E. Dunbar, and R. V. B. Emmons, "Green fluorescent protein retroviral vectors: Low titer and high recombination frequency suggest a selective disadvantage," Human Gene Therapy, vol. 8, no. 11, pp. 1313-1319, 1997.

[39] A. Martinez-Serrano, A. Villa, B. Navarro et al., "Human neural progenitor cells: better blue than green?" Nature Medicine, vol. 6, no. 5, pp. 483-484, 2000.

[40] J. Ji, X. Tong, X. Huang, J. Zhang, H. Qin, and Q. Hu, "Patient-derived human induced pluripotent stem cells from gingival fibroblasts composited with defined nanohydroxyapatite/chitosan/gelatin porous scaffolds as potential bone graft substitutes," Stem Cells Translational Medicine, vol. 5, no. 1, pp. 95-105, 2016.

[41] K. Rutledge, Q. Cheng, M. Pryzhkova, G. M. Harris, and E. Jabbarzadeh, "Enhanced differentiation of human embryonic stem cells on Extracellular matrix-containing Osteomimetic scaffolds for bone tissue engineering," Tissue Engineering-Part C: Methods, vol. 20, no. 11, pp. 865-874, 2014.

[42] J. Liu, W. Chen, Z. Zhao, and H. H. K. Xu, "Reprogramming of mesenchymal stem cells derived from iPSCs seeded on biofunctionalized calcium phosphate scaffold for bone engineering," Biomaterials, vol. 34, no. 32, pp. 7862-7872, 2013.

[43] M. Tang, W. Chen, J. Liu, M. D. Weir, L. Cheng, and H. H. K. Xu, "Human induced pluripotent stem cell-derived mesenchymal stem cell seeding on calcium phosphate scaffold for bone regeneration," Tissue Engineering-Part A, vol. 20, no. 7-8, pp. 1295-1305, 2014.

[44] G. M. de Peppo, I. Marcos-Campos, D. J. Kahler et al., "Engineering bone tissue substitutes from human induced pluripotent stem cells," Proceedings of the National Academy of Sciences of the United States of America, vol. 110, no. 21, pp. 8680-8685, 2013.

[45] D. Marolt, I. M. Campos, S. Bhumiratana et al., "Engineering bone tissue from human embryonic stem cells," Proceedings of the National Academy of Sciences of the United States of America, vol. 109, no. 22, pp. 8705-8709, 2012.

[46] O. H. Jeon, L. M. Panicker, Q. Lu, J. J. Chae, R. A. Feldman, and J. H. Elisseeff, "Human iPSC-derived osteoblasts and osteoclasts together promote bone regeneration in 3D biomaterials," Scientific Reports, vol. 6, article 26761, 2016.

[47] J. Y. Tan, G. Sriram, A. J. Rufaihah, K. G. Neoh, and T. Cao, "Efficient derivation of lateral plate and paraxial mesoderm subtypes from human embryonic stem cells through GSKimediated differentiation," Stem Cells and Development, vol. 22, no. 13, pp. 1893-1906, 2013. 

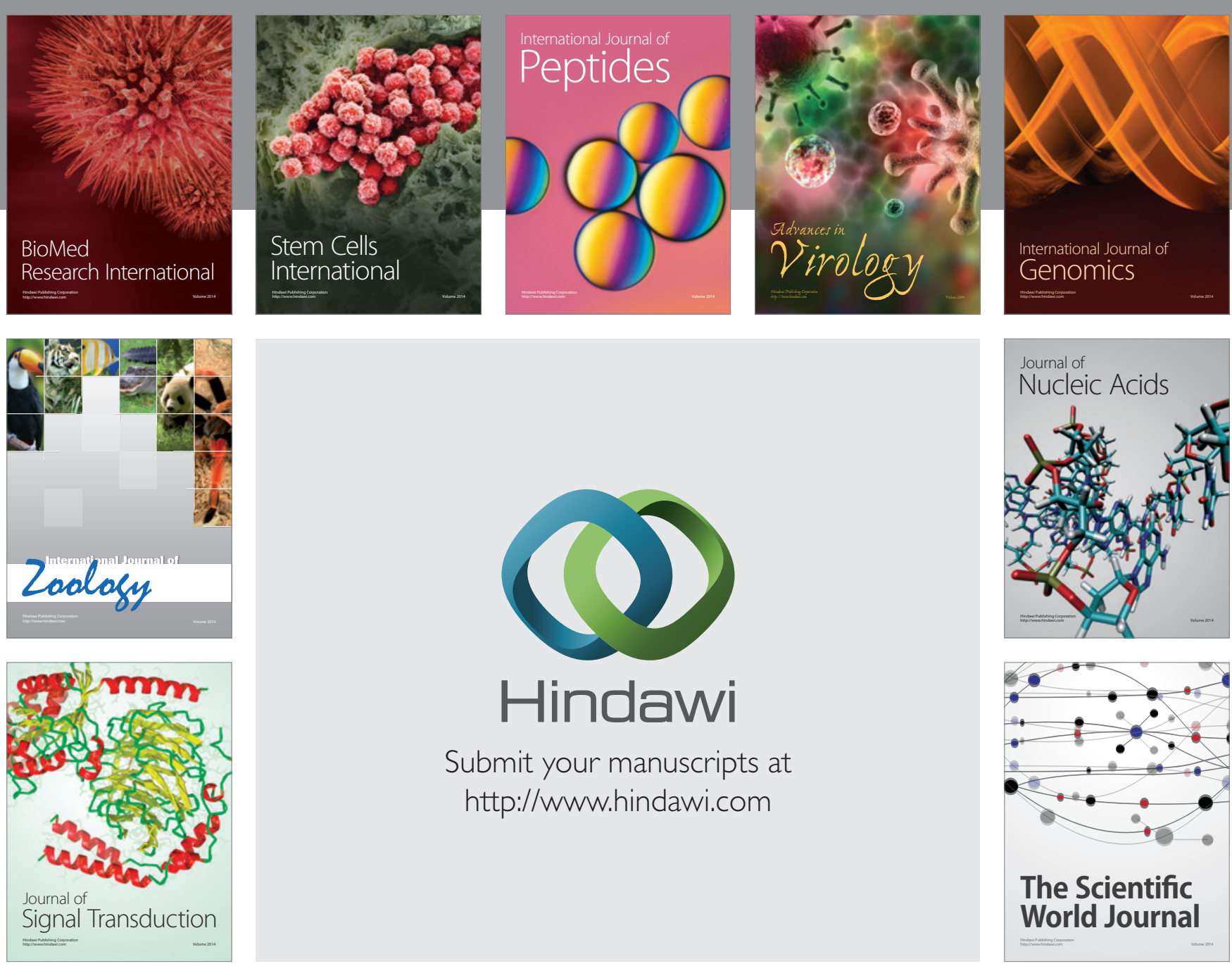

Submit your manuscripts at

http://www.hindawi.com
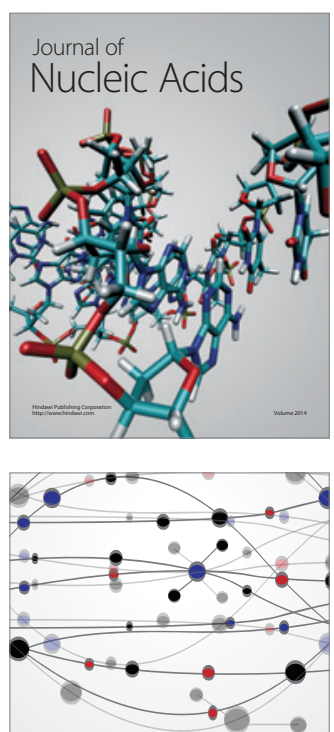

The Scientific World Journal
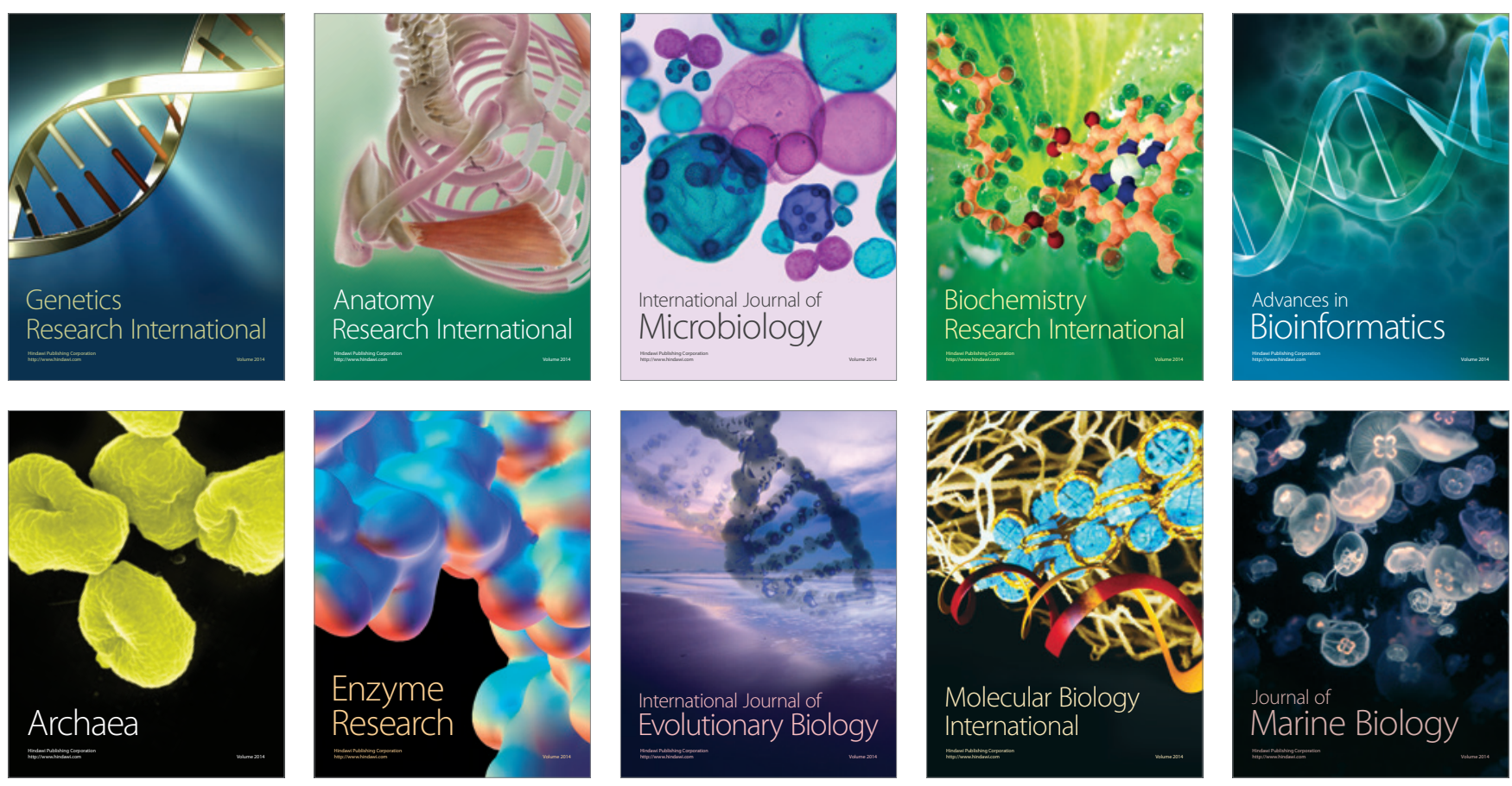